11

\title{
Метод рециркуляции сигналов в задаче наблюдения точечного объекта над металлической поверхностью
}

\author{
(C) В.В. Чапурский, ${ }^{1}$ В.И. Калинин, ${ }^{2}$ А.С. Бугаев, ${ }^{3}$ В.В. Разевиг ${ }^{1}$ \\ ${ }^{1}$ Московский государственный технический университет им. Н.Э. Баумана, \\ 105005 Москва, Россия \\ ${ }^{2}$ Фрязинский филиал Института радиотехники и электроники им. В.А. Котельникова РАН, \\ 141190 Фрязино, Московская обл., Россия \\ ${ }^{3}$ Институт радиотехники и электроники им. В.А. Котельникова РАН, \\ 125009 Москва, Россия \\ e-mail: valch2008@yandex.ru
}

Поступило в Редакцию 12 июля 2018 г.

В окончательной редакции 10 января 2019 г.

Принято к публикации 11 марта 2019 г.

В задаче локализации положения точечного объекта над металлической отражающей поверхностью проанализировано применение сверхширокополосного сигнала в сантиметровом диапазоне длин волн с использованием метода рециркуляции сигналов в кольцах пространственной рециркуляции „излучатель-цель-элемент приемной антенной решетки-излучатель“. Определение координаты объекта над металлической поверхностью основано на вычислении обобщенного корреляционного интеграла в спектральной форме для частотных коэффициентов передачи колец рециркуляции по всем приемным элементам с учетом координат истинного объекта, его зеркального отражения и опорной точки пространства. Дано сопоставление результатов зондирования при наличии и отсутствии рециркуляции.

Ключевые слова: рециркуляция сигнала, обобщенный корреляционный интеграл, антенная решетка, зеркальное отражение, антипод.

DOI: $10.21883 / J T F .2019 .08 .47901 .272-18$

\section{Введение}

Одной из первых работ по рециркуляционным антенным решеткам (АР) была работа [1], а применение таких АР в радиолокации рассматривалось в [2-4]. Теме шумовой радиолокации с рециркуляцией сигналов посвящены работы $[5,6]$. В последние годы интерес к системам с рециркуляцией сигналов и число публикаций по теории и практическим аспектам их применения заметно увеличилось (см., например, [7-9]). Работа [9] посвящена определению обобщенной функции неопределенности по пространственным координатам на основе обобщенного корреляционного интеграла обработки (ОКИ) при использовании метода рециркуляции в мультистатической радиоголографии (МРГ) [10], характеризуемой применением АР. Вместе с тем использование метода рециркуляции в МРГ может иметь ограничения при получении радиоизображений (РИ) многоточечных объектов, исследование которых требует специального анализа.

Наиболее простым в этом плане является случай наблюдения малой неоднородности или точечного объекта в воздухе над идеально отражающей металлической поверхностью. Спецификой такой задачи является наличие зеркального отражения или „антипода“, который расположен симметрично относительно точечного объекта и находится ниже плоскости металлической поверхности. В классическом варианте МРГ наличие ан- типода может приводить к плохому разрешению и смещению координаты истинной отметки в особенности при недостаточной ширине спектра зондирующего сигнала (3C). Несомненный интерес представляет формализация и решение этой задачи в случае применения метода рециркуляции сигналов в кольцах пространственной обратной связи „излучатель-точечная цель-элемент приемной АР-излучатель“. Это обусловлено известным явлением повышения пространственного разрешения при использовании метода рециркуляции $[4,6,9]$.

В настоящей работе рассмотрена микроволновая система получения РИ на основе рециркуляционной антенной системы (AC) при наблюдении точечного рассеивающего объекта в воздухе на фоне антипода, вызванного отражением от металлической поверхности. Предполагается, что объект расположен в воздухе $(\varepsilon=1)$ на произвольной высоте между центром АC, плоскость которой параллельна идеально отражающей бесконечной металлической поверхности, при малых расстояниях между ними, составляющих не более единиц $\mathrm{dm}$. Решение этой частной задачи актуально вследствие того, что метод рециркуляции может быть применен и при зондировании малых неоднородностей над металлической поверхностью в слое диэлектрика с $\varepsilon>1$. Это может иметь практическое значение в задачах диагностики диэлектрических покрытий $[11,12]$, но потребует дополнительных теоретических и экспериментальных исследований. 


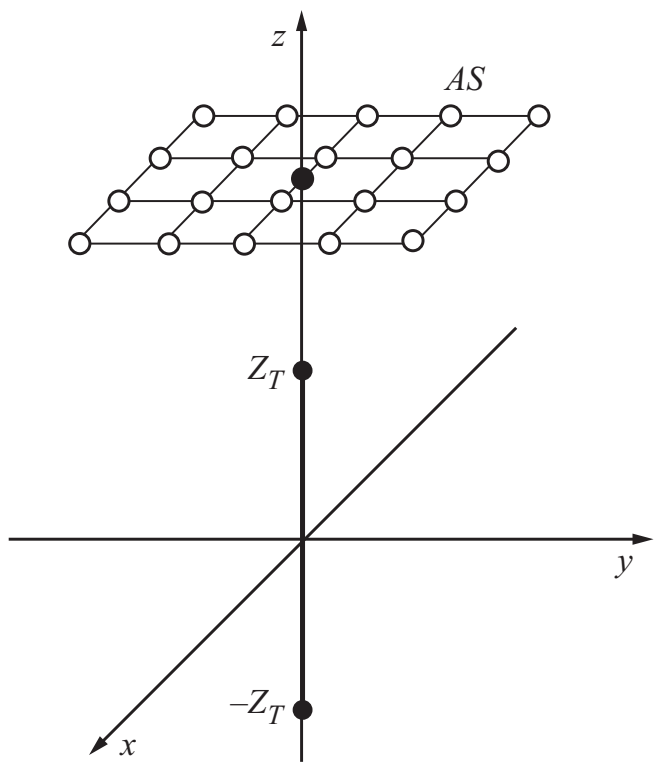

Рис. 1. Вид относительного положения объекта в точке $z_{T}$, его антипода и антенной системы (светлые кружки - приемная АР, черный кружок - излучающий элемент). Металлическая поверхность в плоскости $(x, y, 0)$.

\section{1. Теория наблюдения точечного объекта над металлической поверхностью при методе рециркуляции сигналов}

Рассмотрим АС в виде квадратной приемной АР и одного передающего элемента, например, расположенного в центре приемной АР. Положения АС, точечного объекта и металлической поверхности показаны на рис. 1 в декартовой системе координат $(x, y, z)$. Металлическая поверхность лежит в плоскости $(x, y, 0)$, а точечный объект находится в точке с координатами $\left(0,0, z_{T}\right)$. В условиях идеального отражения от металлической поверхности последняя может быть заменена зеркальным точечным объектом (антиподом) с координатами $\left(0,0,-z_{T}\right)$, также показанным на рис. 1 . В условиях малых расстояний между $\mathrm{AC}$ и металлической поверхностью в качестве зондирующего сигнала (3С) передающего элемента принят сверхширокополосный (СШП) 3С с линейной частотной модуляцией (ЛЧМ).

\section{1. Модель ЛЧМ сигнала передающего элемента AC}

Предположим, что передающий элемент АС излучает на средней частоте $\omega_{0}$ действительный полосовой $3 \mathrm{C}$ $u(t)$ с комплексным спектром $\dot{G}_{u}(\omega)$, которые связаны парой преобразований Фурье. В силу действительности сигнала $u(t)$ его спектр имеет две симметричные ветви на средних частотах $\omega_{0}$ и $-\omega_{0}[13,14]$. В [14] показано, что для таких сигналов, в том числе сверх- широкополосных, остается справедливой традиционная для узкополосных сигналов форма представления в виде комплексного аналитического сигнала $\dot{u}(t)$ :

$$
\dot{u}(t)=\dot{U}(t) \exp \left(j \omega_{0} t\right)
$$

Точка над переменными здесь и далее обозначает комплексные значения. При этом спектры комплексной амплитуды $\dot{U}(t)$ и аналитического сигнала (1) связаны между собой и со смещенной в область низких частот спектральной функцией $\dot{G}_{u}(\omega)$ действительного $3 \mathrm{C} u(t)$ формулами [13]:

$$
\dot{G}_{\dot{U}}(\omega)=\dot{G}_{\dot{u}}\left(\omega_{0}+\omega\right)= \begin{cases}2 \dot{G}_{u}\left(\omega_{0}+\omega\right), & \omega>-\omega_{0}, \\ \dot{G}_{u}\left(\omega_{0}+\omega\right), & \omega=-\omega_{0}, \\ 0, & \omega<-\omega_{0} .\end{cases}
$$

Для ЛЧМ 3С $u(t)$ с девиацией $\Delta \omega=2 \pi \Delta f$ модуль спектра при большой частотно-временной базе на положительных частотах в основном сосредоточен в полосе $\left[\omega_{0}-\Delta \omega / 2, \omega_{0}+\Delta \omega / 2\right][14]$. Тогда при $\omega_{0}>\Delta \omega / 2$ его аналитический сигнал с прямоугольной огибающей $a(t)$ единичной амплитуды и длительности $T$ может быть записан в форме (1), где

$$
\dot{U}(t)=a(t) \exp \left(-j \beta t^{2} / 2\right),
$$

$\beta=2 \pi \gamma, \gamma-$ скорость изменения циклической частоты ЛЧМ ЗС, а частотно-временная база ЛЧМ определена как $B=\Delta f T=\gamma T^{2}$ [14]. Пример нормированного модуля спектра комплексной амплитуды (3) $\dot{U}(t)$ аналитического сигнала представлен на рис. 2 для параметров $T=10^{-7} \mathrm{~s}$, которые соответствуют большой величине частотно-временной базы $B=800$. Спектр рассчитывался с помощью интегралов Френеля и результатов, приведенных в [14,15].

Таким образом, при большой частотно-временной базе ЛЧМ $B \gg 1$, и, в частности, при $B=800$, отвечающей ЛЧМ ЗС для варианта системы с рециркуляцией сигналов, анализируемой далее, модуль спектра Фурье

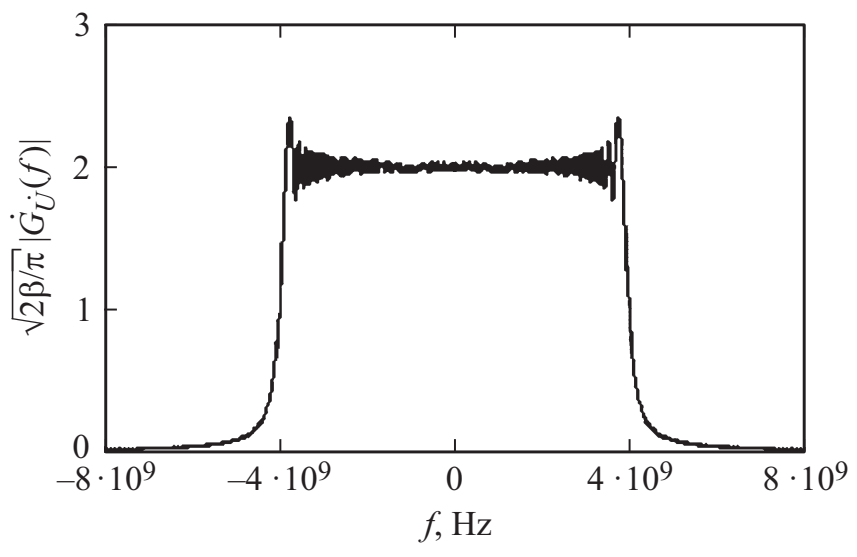

Рис. 2. Нормированный модуль спектра комплексной амплитуды $\dot{U}(t)$ ЛЧМ ЗС при $B=800$. 
$\left|\dot{G}_{\dot{U}}(\omega)\right|$ комплексной огибающей $\dot{U}(t)$ (3) аналитического ЛЧМ ЗС (1) имеет зависимость, близкую к прямоугольной.

\section{2. Обобщенный корреляционный интеграл обработки в системе с рециркуляцией для точечного объекта при наличии антипода}

Представим вектора координат передающего и $N_{r}+1$ приемных элементов АС соответственно в виде $\mathbf{r}_{t}=$ $=\left\|0,0, z_{t}\right\|^{\prime}, \mathbf{r}_{r k}=\left\|x_{r k}, y_{r k}, z_{t}\right\|^{\prime}$, где штрих - символ транспонирования вектора, и $k=0, \ldots, N_{r}$. Произвольную точку пространства в координатах $(x, y, z)$ задаем вектором $\mathbf{R}=\|x, y, z\|^{\prime}$, при котором задержки распространения в кольце пространственной рециркуляции „передающий элемент-точечный объект в точке $\mathbf{R}-k$-й приемный элемент“ равны

$$
\tau_{k}(\mathbf{R})=c^{-1}\left\{\left|\mathbf{R}-\mathbf{r}_{t}\right|+\left|\mathbf{R}-\mathbf{r}_{r k}\right|\right\},
$$

где $c$ - скорость света, $|\mathbf{r}|-$ символ модуля вектора.

В рециркуляционных системах локации принятый отраженный от цели сигнал частично вводится в тракт передачи и в сумме с 3 С передатчика вновь излучается в направлении цели $[1,2]$. При наличии рециркуляции в пространственном кольце обратной связи при одном точечном объекте локации с задержкой распространения $\tau_{k}(\mathbf{R})$ для пары „передающий элемент-объект $-k$-й приемный элемент“" коэффициент передачи кольца рециркуляции при большом, в пределе бесконечном числе рециркуляций с точностью до постоянного множителя, составит $[6,9]$

$$
\dot{K}_{k}(\omega ; \rho, \mathbf{R})=\frac{\exp \left(-j \omega \tau_{k}(\mathbf{R})\right)}{1-\rho \exp \left(-j \omega \tau_{k}(\mathbf{R})\right)} .
$$

В формуле (5) $\rho$ есть коэффициент передачи в пространственной петле положительной обратной связи $(0<\rho<1)$ при отражении сигнала от точечного объекта. Тогда спектр Фурье $\tilde{v}_{k}(j \omega)$ сигнала $v_{k}(t)$ на выходе пространственного рециркулятора равен

$$
\begin{aligned}
\tilde{v}_{k}(j \omega ; \rho, \mathbf{R}) & =\dot{K}_{k}(\omega ; \rho, \mathbf{R}) \tilde{s}(j \omega) \\
& =\dot{K}_{k}(\omega ; \rho, \mathbf{R}) \dot{G}_{U}\left(\omega-\omega_{0}\right),
\end{aligned}
$$

поскольку $\tilde{s}(j \omega)=\dot{G}_{\dot{U}}\left(\omega-\omega_{0}\right)-$ спектр Фурье аналитического ЗС (1).

В рассматриваемом нами случае зеркального отражения от металлической поверхности и наблюдения по рис. 1 имеем реальную и виртуальную точки рассеяния с координатами объекта и антипода

$$
\mathbf{R}_{1}\left(z_{T}\right)=\left\|0,0, z_{T}\right\|^{\prime}, \quad \mathbf{R}_{2}\left(z_{T}\right)=\left\|0,0,-z_{T}\right\|^{\prime} .
$$

При этом результирующие коэффициенты передачи для всех $N_{r}+1$ колец рециркуляции для точечного объекта с координатой $z_{T}$, расположенного над металлической плоскостью, с учетом отражения от объекта и от металлической поверхности составят:

$$
\begin{aligned}
& \dot{K}_{\Sigma k}\left(\omega ; \rho, z_{T}\right)= \\
& \frac{\left\{\dot{q}_{1} \exp \left[-j \omega \tau_{k}\left(\mathbf{R}_{1}\left(z_{T}\right)\right)\right]+\dot{q}_{2} \exp \left[-j \omega \tau_{k}\left(\mathbf{R}_{2}\left(z_{T}\right)\right)\right]\right\}}{1-\rho\left\{\dot{q}_{1} \exp \left[-j \omega \tau_{k}\left(\mathbf{R}_{1}\left(z_{T}\right)\right)\right]+\dot{q}_{2} \exp \left[-j \omega \tau_{k}\left(\mathbf{R}_{2}\left(z_{T}\right)\right)\right]\right\}},
\end{aligned}
$$

где $k=0, \ldots, N_{r}, \dot{q}_{1}$ и $\dot{q}_{2}-$ в общем случае комплексные коэффициенты отражения для истинного и зеркального сигналов, принимаемые при отражении от металлической плоскости одинаковыми по модулю $\left|\dot{q}_{1}\right|=\left|\dot{q}_{2}\right|=q$ и противоположными по фазе $\dot{q}_{1}=q$, $\dot{q}_{2}=q \cdot e^{j \pi}=-q$, а $\rho-$ коэффициент обратной связи в кольце рециркуляции.

Для опорной виртуальной цели с координатой $z_{0}$ будем иметь коэффициент передачи кольца рециркуляции $\dot{K}_{0 k}\left(\omega ; \rho_{0}, z_{0}\right)$, задаваемый формулой $(5)$ в общем случае при ином $\rho=\rho_{0}$ и использовании вместо $\mathbf{R}$ опорного вектора $\mathbf{R}\left(z_{0}\right)=\left\|0,0, z_{0}\right\|^{\prime}$. Соответственно вместо (6) следует записать

$$
\begin{aligned}
\tilde{v}\left(j \omega ; \rho_{0}, z_{0}\right) & =\dot{K}_{0 k}\left(\omega ; \rho_{0}, z_{0}\right) \tilde{s}(j \omega) \\
& =\dot{K}_{0 k}\left(\omega ; \rho_{0}, z_{0}\right) \dot{G}_{\dot{U}}\left(\omega-\omega_{0}\right) .
\end{aligned}
$$

При этом ОКИ в спектральной форме [6,9] для спектров Фурье $\tilde{\nu}_{\Sigma k}\left(j \omega ; \rho, z_{T}\right)$ и $\tilde{v}_{k}\left(j \omega ; \rho_{0}, z_{0}\right)$ с учетом суммирования по всем элементам приемной АР приобретает следующий вид:

$$
\begin{aligned}
\dot{Q}\left(z_{0}, z_{T}\right)= & Q_{\max }^{-1} \sum_{k=0}^{N_{r}} \int_{-\infty}^{\infty} \dot{K}_{\Sigma k}\left(\omega-\omega_{0} ; \rho, z_{T}\right) \\
& \times \dot{K}_{0 k}^{*}\left(\omega-\omega_{0} ; \rho_{0}, z_{0}\left|\dot{G}_{\dot{U}}(\omega)\right|^{2}\right) d \omega,
\end{aligned}
$$

где * - символ комплексного сопряжения, $Q_{\max }^{-1}-$ нормирующий множитель, обеспечивающий максимальное значение модуля $\left|\dot{Q}\left(z_{T}, z_{T}\right)\right|$, равное единице. Приближенное интегрирование в (10) выполнялось численно в пределах полосы частот $|\omega| \leq \Delta \omega$, вне которой модуль спектра $\left|\dot{G}_{\dot{U}}(\omega)\right|$ близок к нулю (рис. 2). Представленный результат (10) с учетом коэффициентов передачи колец рециркуляции (5) и (8), спектра 3С (2), выражений для задержек (4) и значений векторов положения элементов АC $\mathbf{r}_{t}$ и $\mathbf{r}_{r k}$ позволяет рассчитать зависимости модуля ОКИ (10) $\left|\dot{Q}\left(z_{0}, z_{T}\right)\right|$ в функции $z_{0}$ при фиксированном $z_{T}$. На основании рассчитанной зависимости возможна оценка разрешающей способности при заданном положении точечного объекта $z_{T}$ в присутствии зеркального переотражения при использовании метода рециркуляции сигналов и заданных значениях истинного и опорного коэффициентов обратной связи $\rho$ и $\rho_{0}$ пространственных колец рециркуляции.

В отсутствие рециркуляции имеет место случай классической радиоголографии [10]. При этом ОКИ в условиях приема суммы сигналов от реальной и зеркальной 
точечных целей определялся на основании известной методики $[10]$ при $P=1$ и опорном сигнале, соответствующем одиночной виртуальной цели с опорным вектором координат $\mathbf{R}\left(z_{0}\right)=\left\|0,0, z_{0}\right\|^{\prime}$ :

$$
\begin{aligned}
\dot{Q}\left(z_{0}, z_{T}\right)= & Q_{\max }^{-1} \sum_{k=0}^{N_{r}} \int_{-\infty}^{\infty} \sum_{p=0}^{P} \dot{q}_{p} \exp \left[j\left(\omega+\omega_{0}\right)\right. \\
& \left.\times \Delta \tau_{p}\left(z_{0}, z_{T}, k\right)\right]\left|\dot{G}_{\dot{U}}(\omega)\right|^{2} d \omega,
\end{aligned}
$$

где $P=1, \Delta \tau_{p}\left(z_{0}, z_{T}, k\right)=\tau_{k}\left(\mathbf{R}\left(z_{0}\right)\right)-\tau_{k}\left(\mathbf{R}_{p}\left(z_{T}\right)\right)$ при $p=0,1, \quad \tau_{k}(\mathbf{R})$ дается формулой (4), а $\mathbf{R}_{1}\left(z_{T}\right)$ и $\mathbf{R}_{2}\left(z_{T}\right)$ - формулами (7). В приближении прямоугольной формы спектра интегрирование по частоте в (11) выполняется аналитически, и ОКИ в случае классической радиоголографии приобретает вид

$$
\begin{aligned}
\dot{Q}\left(z_{0}, z_{T}\right) \approx & Q_{\max }^{-1} \sum_{k=0}^{N_{r}} \sum_{p=0}^{P} \dot{q}_{p} \exp \left[j \omega_{0} \Delta \tau_{p}\left(z_{0}, z_{T}, k\right)\right] \\
& \times \operatorname{sinc}\left[\frac{\Delta \omega}{2} \Delta \tau_{p}\left(z_{0}, z_{T}, k\right)\right] .
\end{aligned}
$$

Формулы (10)-(12) позволяют производить сравнение разрешающей способности РИ, полученных при классической радиоголографии и при методе рециркуляции в задаче наблюдения точечного объекта над металлической поверхностью. Соответствующие количественные результаты приводятся в примерах разд. 2 .

\section{2. Примеры фокусировки при наличии и отсутствии рециркуляции}

Анализировались примеры систем зондирования с наличием и отсутствием рециркуляции, содержащих АС в виде одного передающего элемента и приемной эквидистантной АР. Рассматривался случай малого расстояния от АC до металлической поверхности, между которыми находился точечный объект. Размеры приемной АР выбраны сопоставимыми с расстоянием от АР до металлической поверхности, что теоретически улучшает избирательность по глубине залегания при зондировании. Параметры системы одинаковые для датчика на основе рециркуляции сигналов и на основе метода классической радиоголографии были следующими:

— средняя частота ЛЧМ ЗС $16 \mathrm{GHz}$

- девиация ЛЧМ сигнала $8 \mathrm{GHz}$;

- число передающих элементов 1 ;

- размеры приемной эквидистантной АР $7 \times 7 \mathrm{~cm}$;

— число элементов приемной АР $5 \times 5$;

- расстояние плоскости АС от металлической поверхности $10 \mathrm{~cm}$.

\section{1. Фокусировка при наличии рециркуляции}

При реализации метода рециркуляции особую роль играет выбор величины коэффициентов положительной обратной связи $\rho<1$ и $\rho_{0}<1$. Этот выбор зависит от интенсивности принимаемых сигналов $q_{1}$ и $q_{2}$, которые входят в выражения для коэффициентов передачи колец рециркуляции в (10) с учетом (5) и (8). Задание $\rho$ и $\rho_{0}$ не должно приводить к появлению сингулярности модулей коэффициентов передачи во всем интервале значений $z_{T}$ и $z_{0}$ вследствие равенства нулю знаменателей в (5) и (8). В частности, выбранные при расчетах значения $q_{1}=q_{2}=1.0$ и $\rho_{0}=0.65, \rho=0.4$ удовлетворяли данному условию в интервалах значений $\left|z_{0}\right|<10 \mathrm{~cm}$ и $\left|z_{T}\right|<10 \mathrm{~cm}$ при исходных данных рассмотренной системы зондирования.

Результаты расчета РИ объекта в виде зависимостей квадрата модуля ОКИ от вертикальной координаты $z_{0}$ при наличии рециркуляции даны на рис. 3 при трех значениях координаты $z_{T}$ точечного объекта. Зависимости получены по формуле (10), учитывающей точную форму модуля спектра $\left|\dot{G}_{U}(\omega)\right|$. Отметим, что дополнительно проводившееся сопоставление с результатами, рассчитанными при прямоугольной аппроксимации модуля спектра, показало идеальное совпадение с результатами, полученными при точной форме модуля спектра
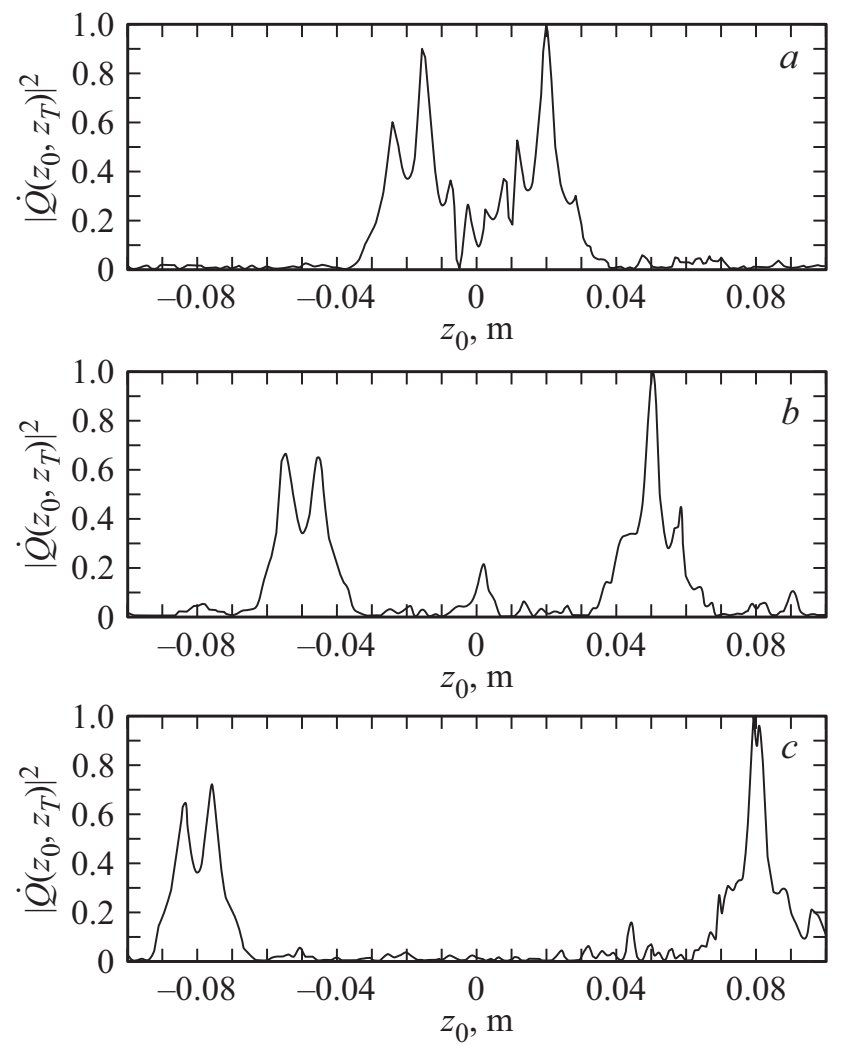

Рис. 3. Зависимости нормированного квадрата модуля ОКИ от опорной координаты $z_{0}$ при методе рециркуляции для $\rho_{0}=0.65, \rho=0.4$ и истинных координатах объекта $z_{T}=2(a)$, $5(b)$ и $8 \mathrm{~cm}(c)$. 
в области положительных значений $z_{0}>0$, и малые, практически не значимые, отличия в области антипода при $z_{0}<0$. Из представленных зависимостей следует, что имеет место заметное подавление лепестка РИ на координате зеркального объекта, в то время как главный лепесток РИ сохраняет свое максимальное значение и с высокой точностью соответствует истинной координате $z_{T}$. Ширина этого лепестка по условному уровню 0.7 , определяющая разрешающую способность и точность измерения координаты $z_{T}$, составляет менее $0.5 \mathrm{~cm}$.

\section{2. Фокусировка методом классической радиоголографии}

В случае классической радиоголографии (при отсутствии рециркуляции) зависимости $\left|\dot{Q}\left(z_{0}, z_{T}\right)\right|^{2}$ от $z_{0}$ в интервале $\left|z_{0}\right| \leq 10 \mathrm{~cm}$ показаны на рис. 4 для двух значений положения точечного объекта $z_{T}=2 \mathrm{~cm}$ и $z_{T}=5 \mathrm{~cm}$. На рис. 4, $a$ при $z_{T}=2 \mathrm{~cm}$ положения максимумов нормированного модуля ОКИ незначительно смещены в сторону металлической поверхности. Это обусловлено взаимным влиянием прямого и зеркального изображений и их боковых лепестков при малом расстоянии $z_{T}$ точечного объекта до поверхности. При увеличении расстояния $z_{T} \geq 5 \mathrm{~cm}$ (рис. $4, b$ ), как показали дополнительные расчеты, смещение максимума главного лепестка изображения относительно истинного значения практически отсутствует.

В целом на основании результатов, полученных при классической радиоголографии, т.е. в отсутствие рециркуляции видно, что результаты фокусировки имеют максимумы, вполне соответствующие как истинному положению объекта, так и его зеркальному отражению. Однако ширина главных лепестков по условному уров-
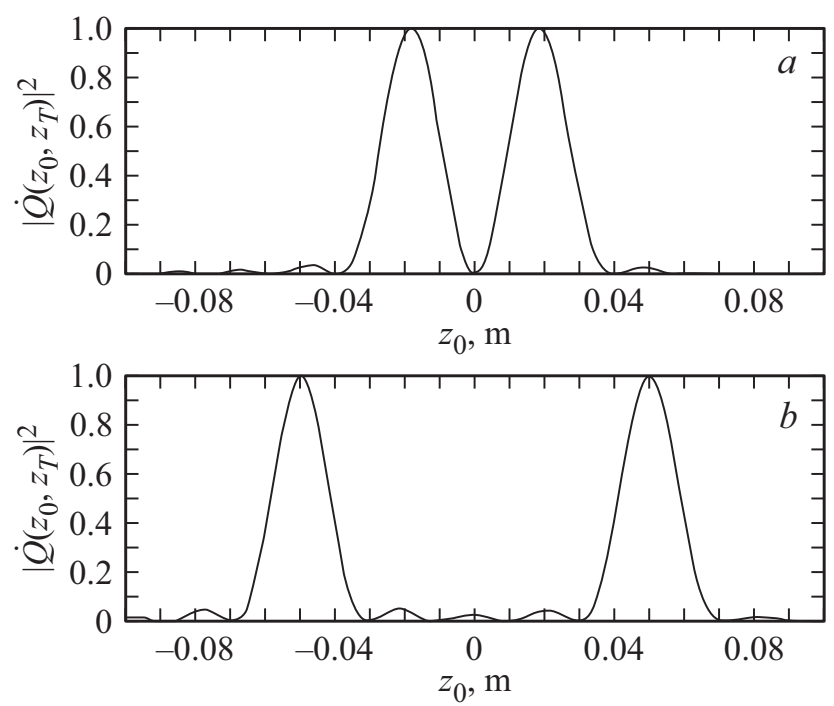

Рис. 4. Зависимость нормированного квадрата модуля ОКИ в случае классической радиоголографии от опорной координаты $z_{0}$ при истинной координате объекта $z_{T}=2(a), 5 \mathrm{~cm}(b)$. ню 0.7 в данном случае более чем в 3 раза превосходит ширину главного лепестка при наличии рециркуляции.

\section{Заключение}

Поставлена и теоретически решена задача наблюдения точечного объекта на фоне отражения от металлической поверхности при использовании метода рециркуляции сигналов. Рассмотрен случай антенной системы, состоящей из одиночного передающего элемента и эквидистантной приемной антенной решетки (AP). При осуществлении режима рециркуляции сверхширокополосного ЛЧМ сигнала в пространственных кольцах обратной связи „излучающий элемент-цель-индивидуальный приемный элемент АР-излучающий элемент“ получено выражение для обобщенного корреляционного интеграла обработки, учитывающее зеркальное отражение точечного объекта от металлической поверхности. Построение радиоизображения в виде квадрата модуля корреляционного интеграла при методе рециркуляции сигналов показало возможность получения высокого разрешения по координате объекта, достигающего единиц миллиметров, а также наличие заметного подавления зеркального максимума РИ.

На конкретном примере зондирования при высоте плоскости АС над металлической поверхностью, равной $10 \mathrm{~cm}$, проведено сравнение результатов, полученных методом рециркуляции сигналов, с методом классической радиоголографии при одинаковых пространственно-частотных параметрах антенной системы, зондирующих сигналов и положениях объектов над металлической поверхностью. Получено, что разрешающая способность системы на основе метода рециркуляции более чем в 3 раза превосходит аналогичную характеристику при методе классической радиоголографии. При этом отсутствует значимое смещение максимума истинного РИ относительно точечного объекта в рассмотренном интервале высот точечного объекта над металлической поверхностью, а также обнаружен эффект заметного подавления РИ зеркального отражения, отсутствующий при методе классической радиоголографии.

Дальнейшие теоретические, а также экспериментальные исследования могут быть направлены на рассмотрение и анализ вариантов систем построения радиоизображений на основе метода рециркуляции сигналов при зондировании точечных неоднородностей, расположенных над металлической поверхностью в диэлектрическом слое с заданной диэлектрической проницаемостью применительно к задачам диагностики диэлектрических покрытий металлических объектов $[11,12]$. Также представляют интерес вопросы выбора иных вариантов антенных систем, ориентированных на конкретные практические задачи. 


\section{Финансирование работы}

Исследование выполнено при поддержке гранта Российского научного фонда (проект № 15-19-00126).

\section{Конфликт интересов}

Авторы заявляют, что у них нет конфликта интересов.

\section{Список литературы}

[1] Pon C.Y. // IEEE Transactions on Antennas and Propagation. 1964. N 2. March. P. 176-180.

[2] Морская радиолокация / Под ред. В.И. Винокурова. Л.: Судостроение, 1986. С. 196-200.

[3] Калинин В.И., Кузмичев В.Е., Мясин Е.А. и др. АС. № 792183. Бюлл. изобретений № 48. 1980.

[4] Калинкевич А.А., Крылова М.С., Турыгин М.С. // Сборник докладов Всероссийской научной конференции „Сверхширокополосные сигналы в радиолокации, связи и акустике“. Муром. 1-3 июля 2003. С. 415-419.

[5] Gupta Shalab, Brown T.R. // IEEE Transactions on Aerospace and Electronic Systems. 2007. Vol. 43. N 2. April. P. 472-479.

[6] Калинин В.И., Чапурский В.В. // РЭ. 2008. Т. 53. Вып. 10. C. $1266-1277$.

[7] Buchanan N.B., Fusco V. // Proceedings of the 7th European Radar Conference. 30 September-1 October 2010. Paris. France. P. 133-136.

[8] Hallbjörner P., Cheng S. // IEEE Antennas and Wireless Propagation Letters. 2013. Vol. 12. P. 1085-1088.

[9] Калинин В.И., Чапурский В.В. // Журнал радиоэлектроники [электронный журнал] 2018. № 4. Режим доступа: http://jre.cplire.ru/jre/apr18/9/text.pdf DOI: $10.30898 / 1684-1719.2018 .4 .9$

[10] Чапурский В.В. // Вестник МГТУ им. Н.Э. Баумана Сер. Приборостроение. 2011. Вып. 4. С. 72-91.

[11] Ивашов С.И., Разевиг В.В., Васильев И.А., Шитиков В.С. // Контроль. Диагностика. 2014. Вып. 12. С. 52-61.

[12] Ивашов С.И., Бугаев А.С., Журавлев А.В., Разевиг В.В., Чижс М.А., Ивашов А.И. // ЖТФ. 2018. Т. 88. Вып. 2. C. $268-275$.

[13] Евсиков Ю.А., Чапурский В.В. Преобразование случайных процессов в радиотехнических устройствах. М.: Высшая школа, 1977. 264 с.

[14] Гоноровский И.С. Радиотехнические цепи и сигналы. Ч. 1. М.: Советское радио, 1966. $608 \mathrm{c}$.

[15] Boersma J. // Math. Comp. 1960. Vol. 14. N 380. 